Research Article

\title{
Differential Subordinations for Certain Meromorphically Multivalent Functions Defined by Dziok-Srivastava Operator
}

\author{
Ying Yang, ${ }^{1}$ Yu-Qin Tao, ${ }^{1}$ and Jin-Lin Liu ${ }^{2}$ \\ ${ }^{1}$ Department of Mathematics, Maanshan Teacher's College, Maanshan 243000, China \\ 2 Department of Mathematics, Yangzhou University, Yangzhou 225002, China
}

Correspondence should be addressed to Jin-Lin Liu, jlliu@yzu.edu.cn

Received 21 January 2011; Revised 14 April 2011; Accepted 20 April 2011

Academic Editor: Malisa R. Zizovic

Copyright (C) 2011 Ying Yang et al. This is an open access article distributed under the Creative Commons Attribution License, which permits unrestricted use, distribution, and reproduction in any medium, provided the original work is properly cited.

By making use of the Dziok-Srivastava operator, we introduce a new class of meromorphically multivalent functions. Some inclusion properties of functions belonging to this class are derived.

\section{Introduction}

Let $\Sigma(p)$ denote the class of functions of the form

$$
f(z)=z^{-p}+\sum_{n=1}^{\infty} a_{n} z^{n-p} \quad(p \in \mathbb{N}:=\{1,2,3, \ldots\}),
$$

which are analytic in the punctured open unit disk $\mathbb{U}_{0}=\{z: 0<|z|<1\}$ with a pole at $z=0$. Also let the Hadamard product (or convolution) of the following functions:

$$
f_{j}(z)=z^{-p}+\sum_{n=1}^{\infty} a_{n, j} z^{n-p} \quad(j=1,2)
$$

be given by

$$
\left(f_{1} * f_{2}\right)(z):=z^{-p}+\sum_{n=1}^{\infty} a_{n, 1} a_{n, 2} z^{n-p}=\left(f_{2} * f_{1}\right)(z) .
$$


Given two functions $f(z)$ and $g(z)$, which are analytic in $\mathbb{U}=\mathbb{U}_{0} \cup\{0\}$, we say that the function $g(z)$ is subordinate to $f(z)$ and write $g \prec f$ or (more precisely) $g(z) \prec f(z)(z \in \mathbb{U})$, if there exists a Schwarz function $w(z)$, analytic in $\mathbb{U}$ with $w(0)=0$ and $|w(z)|<1(z \in$ $\mathbb{U})$ such that $g(z)=f(w(z))(z \in \mathbb{U})$. In particular, if $f(z)$ is univalent in $\mathbb{U}$, we have the following equivalence:

$$
g(z) \prec f(z) \quad(z \in \mathbb{U}) \Longleftrightarrow g(0)=f(0), \quad g(\mathbb{U}) \subset f(\mathbb{U})
$$

Let $A$ be the class of functions of the form

$$
f(z)=z+\sum_{n=2}^{\infty} b_{n} z^{n}
$$

which are analytic in $\mathbb{U}$. A function $f(z) \in A$ is said to be in the class $S^{*}(\alpha)$ if

$$
\operatorname{Re}\left\{\frac{z f^{\prime}(z)}{f(z)}\right\}>\alpha \quad(z \in \mathbb{U})
$$

for some $\alpha(\alpha<1)$. When $0 \leq \alpha<1, S^{*}(\alpha)$ is the class of starlike functions of order $\alpha$ in $\mathbb{U}$. A function $f(z) \in A$ is said to be prestarlike of order $\alpha$ in $\mathbb{U}$ if

$$
\frac{z}{(1-z)^{2(1-\alpha)}} * f(z) \in S^{*}(\alpha) \quad(\alpha<1)
$$

where the symbol $*$ means the familiar Hadamard product (or convolution) of two analytic functions in $\mathbb{U}$. We denote this class by $R(\alpha)$ (see [1]). Clearly a function $f(z) \in A$ is in the class $R(0)$ if and only if $f(z)$ is convex univalent in $\mathbb{U}$ and $R(1 / 2)=S^{*}(1 / 2)$.

For complex parameters

$$
\alpha_{1}, \ldots, \alpha_{q} \text { and } \beta_{1}, \ldots, \beta_{s} \quad\left(\beta_{j} \notin \mathbb{Z}_{0}^{-}:=\{0,-1,-2, \ldots\} ; j=1,2, \ldots, s\right) \text {, }
$$

we define the generalized hypergeometric function ${ }_{q} F_{s}\left(\alpha_{1}, \ldots, \alpha_{q} ; \beta_{1}, \ldots, \beta_{s} ; z\right)$ by

$$
\begin{array}{r}
{ }_{q} F_{s}\left(\alpha_{1}, \ldots, \alpha_{q} ; \beta_{1}, \ldots, \beta_{s} ; z\right):=\sum_{n=0}^{\infty} \frac{\left(\alpha_{1}\right)_{n} \cdots\left(\alpha_{q}\right)_{n}}{\left(\beta_{1}\right)_{n} \cdots\left(\beta_{s}\right)_{n}} \cdot \frac{z^{n}}{n !} \\
\left(q \leq s+1 ; q, s \in \mathbb{N}_{0}:=\mathbb{N} \cup\{0\} ; z \in \mathbb{U}\right),
\end{array}
$$

where $(x)_{n}$ is the Pochhammer symbol defined, in terms of the Gamma function $\Gamma(z)$,by

$$
(x)_{n}:=\frac{\Gamma(x+n)}{\Gamma(x)}= \begin{cases}1 & (n=0 ; x \in \mathbb{C} \backslash\{0\}) \\ x(x+1) \cdots(x+n-1) & (n \in \mathbb{N} ; x \in \mathbb{C}) .\end{cases}
$$


Corresponding to a function $h_{p}\left(\alpha_{1}, \ldots, \alpha_{q} ; \beta_{1}, \ldots, \beta_{s} ; z\right)$ defined by

$$
h_{p}\left(\alpha_{1}, \ldots, \alpha_{q} ; \beta_{1}, \ldots, \beta_{s} ; z\right)=z^{-p} F_{s}\left(\alpha_{1}, \ldots, \alpha_{q} ; \beta_{1}, \ldots, \beta_{s} ; z\right) \text {, }
$$

we now consider a linear operator

$$
H_{p}\left(\alpha_{1}, \ldots, \alpha_{q} ; \beta_{1}, \ldots, \beta_{s}\right): \Sigma(p) \longrightarrow \Sigma(p),
$$

defined by means of the Hadamard product (or convolution) as follows:

$$
H_{p}\left(\alpha_{1}, \ldots, \alpha_{q} ; \beta_{1}, \ldots, \beta_{s}\right) f(z):=h_{p}\left(\alpha_{1}, \ldots, \alpha_{q} ; \beta_{1}, \ldots, \beta_{s} ; z\right) * f(z)
$$

For convenience, we write

$$
H_{p, q, s}\left(\alpha_{1}\right):=H_{p}\left(\alpha_{1}, \ldots, \alpha_{q} ; \beta_{1}, \ldots, \beta_{s}\right)
$$

Thus, after some calculations, we have

$$
z\left(H_{p, q, s}\left(\alpha_{1}\right) f(z)\right)^{\prime}=\alpha_{1} H_{p, q, s}\left(\alpha_{1}+1\right) f(z)-\left(\alpha_{1}+p\right) H_{p, q, s}\left(\alpha_{1}\right) f(z) .
$$

The operator $H_{p, q, s}\left(\alpha_{1}\right)$ is popularly known as the generalized Dziok-Srivastava operator. Many interesting subclasses of multivalent functions, associated with the operator $H_{p, q, s}\left(\alpha_{1}\right)$ and its various special cases, were investigated recently by (e.g.) Dziok and Srivastava [2-4], Liu [5], Liu and Srivastava [6, 7], Patel et al. [8], Wang et al. [9], and others.

Let $P$ be the class of functions $h(z)$ with $h(0)=1$, which are analytic and convex univalent in $\mathbb{U}$.

Definition 1.1. A function $f(z) \in \Sigma(p)$ is said to be in the class $T_{p, q, s}\left(\alpha_{1}, \lambda ; h\right)$ if it satisfies the subordination condition

$$
\frac{(\lambda-1)}{p} z^{p+1}\left(H_{p, q, s}\left(\alpha_{1}\right) f(z)\right)^{\prime}+\frac{\lambda}{p(p+1)} z^{p+2}\left(H_{p, q, s}\left(\alpha_{1}\right) f(z)\right)^{\prime \prime} \prec h(z)
$$

where $\lambda$ is a complex number and $h(z) \in P$.

The main object of this paper is to present a systematic investigation of the class $T_{p, q, s}\left(\alpha_{1}, \lambda ; h\right)$ defined above by means of the generalized Dziok-Srivastava operator $H_{p, q, s}\left(\alpha_{1}\right)$.

For our purpose, we shall need the following lemmas to derive our main results for the class $T_{p, q, s}\left(\alpha_{1}, \lambda ; h\right)$.

Lemma 1.2 (see [10]). Let $g(z)$ be analytic in $\mathbb{U}$ and $h(z)$ be analytic and convex univalent in $\mathbb{U}$ with $h(0)=g(0)$. If

$$
g(z)+\frac{1}{\mu} z g^{\prime}(z) \prec h(z)
$$


where $\operatorname{Re} \mu>0$, then

$$
g(z) \prec \tilde{h}(z)=\mu z^{-\mu} \int_{v}^{z} t^{\mu-1} h(t) d t \prec h(z)
$$

and $\tilde{h}(z)$ is the best dominant of (1.17).

Lemma 1.3 (see [1]). Let $\alpha<1, f(z) \in S^{*}(\alpha)$ and $g(z) \in R(\alpha)$. Then, for any analytic function $F(z)$ in $\mathbb{U}$,

$$
\frac{g *(f F)}{g * f}(\mathbb{U}) \subset \overline{\mathrm{co}}(F(\mathbb{U}))
$$

where $\overline{\mathrm{co}}(F(\mathbb{U}))$ denotes the closed convex hull of $F(\mathbb{U})$.

\section{Properties of the Class $T_{p, q, s}\left(\alpha_{1}, \lambda ; h\right)$}

Theorem 2.1. Let $\lambda_{1}<\lambda_{2} \leq 0$. Then $T_{p, q, s}\left(\alpha_{1}, \lambda_{1} ; h\right) \subset T_{p, q, s}\left(\alpha_{1}, \lambda_{2} ; h\right)$.

Proof. Let $\lambda_{1}<\lambda_{2} \leq 0$ and suppose that

$$
g(z)=-\frac{z^{p+1}\left(H_{p, q, s}\left(\alpha_{1}\right) f(z)\right)^{\prime}}{p}
$$

for $f(z) \in T_{p, q, s}\left(\alpha_{1}, \lambda_{1} ; h\right)$. Then the function $g(z)$ is analytic in $\mathbb{U}$ with $g(0)=1$. Differentiating both sides of (2.1) with respect to $z$ and using (1.16), we have

$$
\frac{\left(\lambda_{1}-1\right)}{p} z^{p+1}\left(H_{p, q, s}\left(\alpha_{1}\right) f(z)\right)^{\prime}+\frac{\lambda_{1}}{p(p+1)} z^{p+2}\left(H_{p, q, s}\left(\alpha_{1}\right) f(z)\right)^{\prime \prime}=g(z)-\frac{\lambda_{1}}{p+1} z g^{\prime}(z) \prec h(z) .
$$

Hence an application of Lemma 1.2 yields

$$
g(z) \prec h(z) .
$$

Noting that $0<\lambda_{2} / \lambda_{1}<1$ and that $h(z)$ is convex univalent in $\mathbb{U}$, it follows from (2.1) to (2.3) that

$$
\begin{aligned}
& \frac{\left(\lambda_{2}-1\right)}{p} z^{p+1}\left(H_{p, q, s}\left(\alpha_{1}\right) f(z)\right)^{\prime}+\frac{\lambda_{2}}{p(p+1)} z^{p+2}\left(H_{p, q, s}\left(\alpha_{1}\right) f(z)\right)^{\prime \prime} \\
& \quad=\frac{\lambda_{2}}{\lambda_{1}}\left(\frac{\left(\lambda_{1}-1\right)}{p} z^{p+1}\left(H_{p, q, s}\left(\alpha_{1}\right) f(z)\right)^{\prime}+\frac{\lambda_{1}}{p(p+1)} z^{p+2}\left(H_{p, q, s}\left(\alpha_{1}\right) f(z)\right)^{\prime \prime}\right) \\
& \quad+\left(1-\frac{\lambda_{2}}{\lambda_{1}}\right) g(z) \prec h(z) .
\end{aligned}
$$

Thus $f(z) \in T p, q, s\left(\alpha_{1}, \lambda_{2} ; h\right)$ and the proof of Theorem 2.1 is completed. 
Theorem 2.2. Let $0<b_{1}<b_{2}$. Then $T_{p, q, s}\left(b_{2}, \lambda ; h\right) \subset T_{p, q, s}\left(b_{1}, \lambda ; h\right)$.

Proof. Define a function $g(z)$ by

$$
g(z)=z+\sum_{n=1}^{\infty} \frac{\left(b_{1}\right)_{n}}{\left(b_{2}\right)_{n}} z^{n+1} \quad\left(z \in \mathbb{U} ; 0<b_{1}<b_{2}\right) .
$$

Then

$$
z^{p+1} h_{p}\left(b_{1}, \alpha_{2}, \ldots, \alpha_{s}, 1 ; b_{2}, \alpha_{2}, \ldots, \alpha_{s} ; z\right)=g(z) \in A,
$$

where

$$
h_{p}\left(b_{1}, \alpha_{2}, \ldots, \alpha_{s}, 1 ; b_{2}, \alpha_{2}, \ldots, \alpha_{s} ; z\right)
$$

is defined as in (1.11), and

$$
\frac{z}{(1-z)^{b_{2}}} * g(z)=\frac{z}{(1-z)^{b_{1}}}
$$

By (2.8), we see that

$$
\frac{z}{(1-z)^{b_{2}}} * g(z) \in S^{*}\left(1-\frac{b_{1}}{2}\right) \subset S^{*}\left(1-\frac{b_{2}}{2}\right) \quad\left(0<b_{1}<b_{2}\right),
$$

which implies that

$$
g(z) \in R\left(1-\frac{b_{2}}{2}\right)
$$

Let $f(z) \in T_{p, q, s}\left(b_{2}, \lambda ; h\right)$. It is easy to verify that

$$
\begin{gathered}
z^{p+1}\left(H_{p, q, s}\left(b_{1}\right) f(z)\right)^{\prime}=\left(z^{p} h_{p}\left(b_{1}, \alpha_{2}, \ldots, \alpha_{s}, 1 ; b_{2}, \alpha_{2}, \ldots, \alpha_{s} ; z\right)\right) *\left(z^{p+1}\left(H_{p, q, s}\left(b_{2}\right) f(z)\right)^{\prime}\right) \\
z^{p+2}\left(H_{p, q, s}\left(b_{1}\right) f(z)\right)^{\prime \prime}=\left(z^{p} h_{p}\left(b_{1}, \alpha_{2}, \ldots, \alpha_{s}, 1 ; b_{2}, \alpha_{2}, \ldots, \alpha_{s} ; z\right)\right) *\left(z^{p+2}\left(H_{p, q, s}\left(b_{2}\right) f(z)\right)^{\prime \prime}\right) .
\end{gathered}
$$

From (2.11), (2.12), and (2.6), we deduce that

$$
\begin{aligned}
& \frac{(\lambda-1)}{p} z^{p+1}\left(H_{p, q, s}\left(b_{1}\right) f(z)\right)^{\prime}+\frac{\lambda}{p(p+1)} z^{p+2}\left(H_{p, q, s}\left(b_{1}\right) f(z)\right)^{\prime \prime} \\
& =\frac{g(z)}{z} * w(z)=\frac{g(z) *(z w(z))}{g(z) * z}
\end{aligned}
$$


where

$$
w(z):=\frac{(\lambda-1)}{p} z^{p+1}\left(H_{p, q, s}\left(b_{2}\right) f(z)\right)^{\prime}+\frac{\lambda}{p(p+1)} z^{p+2}\left(H_{p, q, s}\left(b_{2}\right) f(z)\right)^{\prime \prime} \prec h(z) .
$$

Since the function $z$ belongs to the function class $S^{*}\left(1-b_{2} / 2\right)$ and $h(z)$ is convex univalent in $\mathbb{U}$, it follows from (2.12), (2.13), (2.14), and Lemma 1.3 that

$$
\frac{(\lambda-1)}{p} z^{p+1}\left(H_{p, q, s}\left(b_{1}\right) f(z)\right)^{\prime}+\frac{\lambda}{p(p+1)} z^{p+2}\left(H_{p, q, s}\left(b_{1}\right) f(z)\right)^{\prime \prime}<h(z) .
$$

Thus $f(z) \in T_{p, q, s}\left(b_{1}, \lambda ; h\right)$ and the proof of Theorem 2.2 is completed.

Theorem 2.3. Let $f(z) \in T_{p, q, s}\left(\alpha_{1}, \lambda ; h\right), g(z) \in \Sigma(p)$ and

$$
\operatorname{Re}\left\{z^{p} g(z)\right\}>\frac{1}{2} \quad(z \in \mathbb{U}) .
$$

Then

$$
(f * g)(z) \in T_{p, q, s}\left(\alpha_{1}, \lambda ; h\right) .
$$

Proof. For $f(z) \in T_{p, q, s}\left(\alpha_{1}, \lambda ; h\right)$ and $g(z) \in \Sigma(p)$, we have

$$
\begin{aligned}
& \frac{(\lambda-1)}{p} z^{p+1}\left(H_{p, q, s}\left(\alpha_{1}\right)(f * g)(z)\right)^{\prime}+\frac{\lambda}{p(p+1)} z^{p+2}\left(H_{p, q, s}\left(\alpha_{1}\right)(f * g)(z)\right)^{\prime \prime} \\
& \quad=\frac{(\lambda-1)}{p}\left(z^{p} g(z)\right) *\left(z^{p+1}\left(H_{p, q, s}\left(\alpha_{1}\right) f(z)\right)^{\prime}\right)+\frac{\lambda}{p(p+1)}\left(z^{p} g(z)\right) *\left(z^{p+2}\left(H_{p, q, s}\left(\alpha_{1}\right) f(z)\right)^{\prime \prime}\right) \\
& \quad=\left(z^{p} g(z)\right) * \psi(z),
\end{aligned}
$$

where

$$
\psi(z)=\frac{(\lambda-1)}{p} z^{p+1}\left(H_{p, q, s}\left(\alpha_{1}\right) f(z)\right)^{\prime}+\frac{\lambda}{p(p+1)} z^{p+2}\left(H_{p, q, s}\left(\alpha_{1}\right) f(z)\right)^{\prime \prime} .
$$

In view of (2.16), the function $z^{p} g(z)$ has the Herglotz representation

$$
z^{p} g(z)=\int_{|x|=1} \frac{d \mu(x)}{1-x z} \quad(z \in \mathbb{U})
$$

where $\mu(x)$ is a probability measure defined on the unit circle $|x|=1$ and

$$
\int_{|x|=1} d \mu(x)=1
$$


Since $h(z)$ is convex univalent in $\mathbb{U}$, it follows from (2.18) to (2.20) that

$$
\begin{aligned}
& \frac{(\lambda-1)}{p} z^{p+1}\left(H_{p, q, s}\left(\alpha_{1}\right)(f * g)(z)\right)^{\prime}+\frac{\lambda}{p(p+1)} z^{p+2}\left(H_{p, q, s}\left(\alpha_{1}\right)(f * g)(z)\right)^{\prime \prime} \\
& \quad=\int_{|x|=1} \psi(x z) d \mu(x) \prec h(z) .
\end{aligned}
$$

This shows that $(f * g)(z) \in T_{p, q, s}\left(\alpha_{1}, \lambda ; h\right)$ and the theorem is proved.

Theorem 2.4. Let $f(z) \in T_{p, q, s}\left(\alpha_{1}, \lambda ; h\right), g(z) \in \Sigma(p)$ and

$$
z^{p+1} g(z) \in R(\alpha) \quad(\alpha<1)
$$

Then

$$
(f * g)(z) \in T_{p, q, s}\left(\alpha_{1}, \lambda ; h\right)
$$

Proof. For $f(z) \in T_{p, q, s}\left(\alpha_{1}, \lambda ; h\right)$ and $g(z) \in \Sigma(p)$, from (2.18) we have

$$
\begin{aligned}
& \frac{(\lambda-1)}{p} z^{p+1}\left(H_{p, q, s}(f * g)(z)\right)^{\prime}+\frac{\lambda}{p(p+1)} z^{p+2}\left(H_{p, q, s}(f * g)(z)\right)^{\prime \prime} \\
& =\frac{\left(z^{p+1} g(z)\right) *(z \psi(z))}{\left(z^{p+1} g(z)\right) * z} \quad(z \in \mathbb{U}),
\end{aligned}
$$

where $\psi(z)$ is defined as in (2.19).

Since $h(z)$ is convex univalent in $\mathbb{U}$,

$$
\psi(z) \prec h(z), \quad z^{p+1} g(z) \in R(\alpha), \quad z \in S^{*}(\alpha)(\alpha<1),
$$

it follows from (2.25) and Lemma 1.3 the desired result.

Theorem 2.5. Let $\lambda<0, \beta>0$ and $f(z) \in T_{p, q, s}(\lambda ; \beta h+1-\beta)$. If $\beta \leq \beta_{0}$, where

$$
\beta_{0}=\frac{1}{2}\left(1+\frac{p+1}{\lambda} \int_{0}^{1} \frac{u^{-((p+1) / \lambda)-1}}{1+u} d u\right)^{-1}
$$

then $f(z) \in T_{p, q, s}(0 ; h)$. The bound $\beta_{0}$ is sharp when $h(z)=1 /(1-z)$.

Proof. Let us define

$$
g(z)=-\frac{z^{p+1}\left(H_{p, q, s}\left(\alpha_{1}\right) f(z)\right)^{\prime}}{p}
$$


for $f(z) \in T_{p, q, s}(\lambda ; \beta h+1-\beta)$ with $\lambda<0$ and $\beta>0$. Then we have

$$
\begin{aligned}
g(z)-\frac{\lambda}{p+1} z g^{\prime}(z) & =\frac{(\lambda-1)}{p} z^{p+1}\left(H_{p, q, s}\left(\alpha_{1}\right) f(z)\right)^{\prime}+\frac{\lambda}{p(p+1)} z^{p+2}\left(H_{p, q, s}\left(\alpha_{1}\right) f(z)\right)^{\prime \prime} \\
& \prec \beta h(z)+1-\beta .
\end{aligned}
$$

Hence an application of Lemma 1.2 yields

$$
\begin{aligned}
g(z) & \prec-\frac{\beta(p+1)}{\lambda} z^{(p+1) / \lambda} \int_{0}^{z} t^{-((p+1) / \lambda)-1} h(t) d t+1-\beta \\
& =(h * \psi)(z),
\end{aligned}
$$

where

$$
\psi(z)=-\frac{\beta(p+1)}{\lambda} z^{(p+1) / \lambda} \int_{0}^{z} \frac{t^{-((p+1) / \lambda)-1}}{1-t} d t+1-\beta .
$$

If $0<\beta \leq \beta_{0}$, where $\beta_{0}(>1)$ is given by (2.27), then it follows from (2.31) that

$$
\begin{aligned}
\operatorname{Re} \psi(z) & =-\frac{\beta(p+1)}{\lambda} \int_{0}^{1} u^{-((p+1) / \lambda)-1} \operatorname{Re}\left(\frac{1}{1-u z}\right) d u+1-\beta \\
& >-\frac{\beta(p+1)}{\lambda} \int_{0}^{1} \frac{u^{-((p+1) / \lambda)-1}}{1+u} d u+1-\beta \\
& \geq \frac{1}{2} \quad(z \in \mathbb{U} ; \lambda<0) .
\end{aligned}
$$

Now, by using the Herglotz representation for $\psi(z)$, from (2.28) and (2.30), we arrive at

$$
-\frac{z^{p+1}\left(H_{p, q, s}\left(\alpha_{1}\right) f(z)\right)^{\prime}}{p} \prec(h * \psi)(z) \prec h(z)
$$

because $h(z)$ is convex univalent in $\mathbb{U}$. This shows that $f(z) \in T_{p, q, s}(0 ; h)$.

For $h(z)=1 /(1-z)$ and $f(z) \in \Sigma(p)$ defined by

$$
-\frac{z^{p+1}\left(H_{p, q, s}\left(\alpha_{1}\right) f(z)\right)^{\prime}}{p}=-\frac{\beta(p+1)}{\lambda} z^{(p+1) / \lambda} \int_{0}^{z} \frac{t^{-((p+1) / \lambda)-1}}{1-t} d t+1-\beta,
$$

it is easy to verify that

$$
\frac{(\lambda-1)}{p} z^{p+1}\left(H_{p, q, s}\left(\alpha_{1}\right) f(z)\right)^{\prime}+\frac{\lambda}{p(p+1)} z^{p+2}\left(H_{p, q, s}\left(\alpha_{1}\right) f(z)\right)^{\prime \prime}=\beta h(z)+1-\beta .
$$


Thus $f(z) \in T_{p, q, s}(\lambda ; \beta h+1-\beta)$. Also, for $\beta>\beta_{0}$, we have

$$
\operatorname{Re}\left\{-\frac{z^{p+1}\left(H_{p, q, s}\left(\alpha_{1}\right) f(z)\right)^{\prime}}{p}\right\} \rightarrow-\frac{\beta(p+1)}{\lambda} \int_{0}^{1} \frac{u^{-((p+1) / \lambda)-1}}{1+u} d u+1-\beta<\frac{1}{2} \quad(z \longrightarrow-1),
$$

which implies that $f(z) \notin T_{p, q, s}(0 ; h)$. Hence the bound $\beta_{0}$ cannot be increased when $h(z)=$ $1 /(1-z)$.

\section{Acknowledgment}

The authers would like to express sincere thanks to the referees for careful reading and suggestions which helped us to improve the paper.

\section{References}

[1] S. Ruscheweyh, Convolutions in Geometric Function Theory, vol. 83 of Séminaire de Mathématiques Supérieures, Les Presses de l’Université de Montréal, Montréal, Canada, 1982.

[2] J. Dziok and H. M. Srivastava, "Classes of analytic functions associated with the generalized hypergeometric function," Applied Mathematics and Computation, vol. 103, no. 1, pp. 1-13, 1999.

[3] J. Dziok and H. M. Srivastava, "Some subclasses of analytic functions with fixed argument of coefficients associated with the generalized hypergeometric function," Advanced Studies in Contemporary Mathematics, vol. 5, no. 2, pp. 115-125, 2002.

[4] J. Dziok and H. M. Srivastava, "Certain subclasses of analytic functions associated with the generalized hypergeometric function," Integral Transforms and Special Functions, vol. 14, no. 1, pp. 7-18, 2003.

[5] J.-L. Liu, "Strongly starlike functions associated with the Dziok-Srivastava operator," Tamkang Journal of Mathematics, vol. 35, no. 1, pp. 37-42, 2004.

[6] J.-L. Liu and H. M. Srivastava, "Certain properties of the Dziok-Srivastava operator," Applied Mathematics and Computation, vol. 159, no. 2, pp. 485-493, 2004.

[7] J.-L. Liu and H. M. Srivastava, "A class of multivalently analytic functions associated with the DziokSrivastava operator," Integral Transforms and Special Functions, vol. 20, no. 5, pp. 401-417, 2009.

[8] J. Patel, A. K. Mishra, and H. M. Srivastava, "Classes of multivalent analytic functions involving the Dziok-Srivastava operator," Computers E Mathematics with Applications, vol. 54, no. 5, pp. 599-616, 2007.

[9] Z.-G. Wang, Y.-P. Jiang, and H. M. Srivastava, "Some subclasses of multivalent analytic functions involving the Dziok-Srivastava operator," Integral Transforms and Special Functions, vol. 19, no. 2, pp. 129-146, 2008.

[10] S. S. Miller and P. T. Mocanu, "Differential subordinations and univalent functions," The Michigan Mathematical Journal, vol. 28, no. 2, pp. 157-172, 1981. 


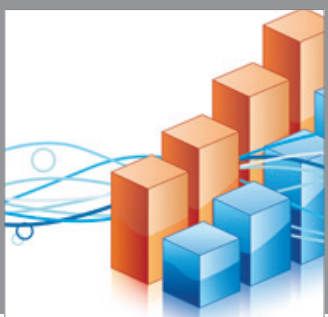

Advances in

Operations Research

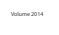

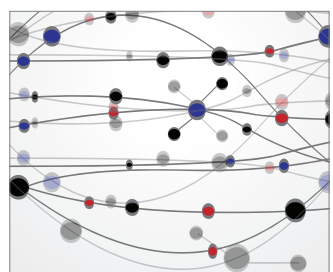

\section{The Scientific} World Journal
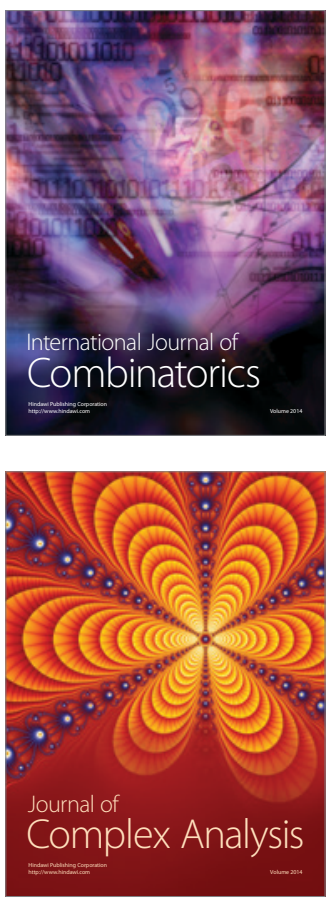

International Journal of

Mathematics and

Mathematical

Sciences
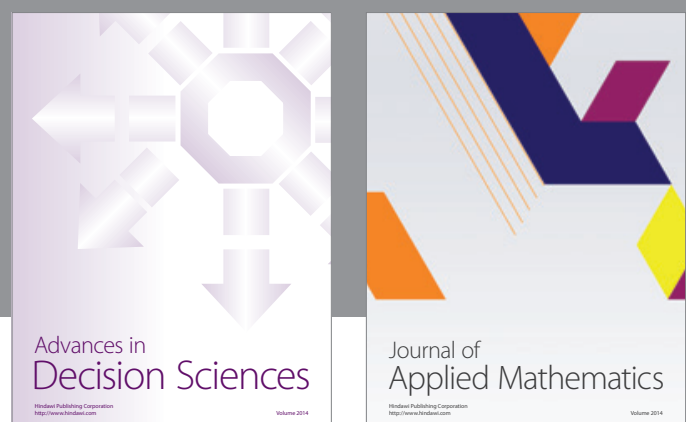

Journal of

Applied Mathematics
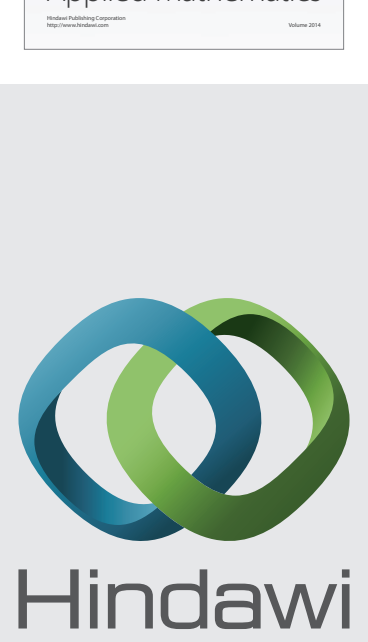

Submit your manuscripts at http://www.hindawi.com
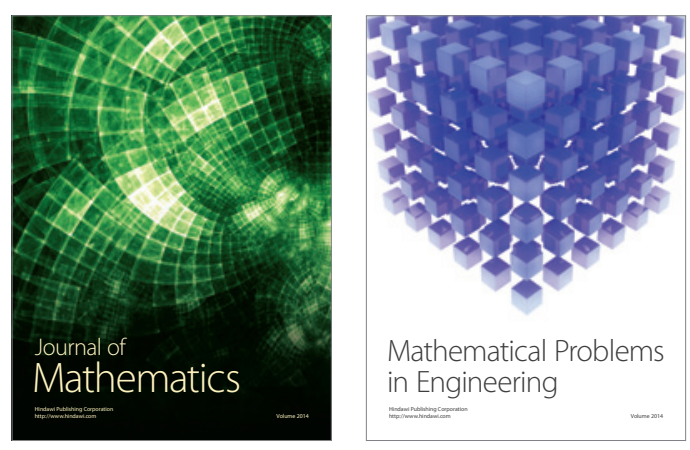

Mathematical Problems in Engineering
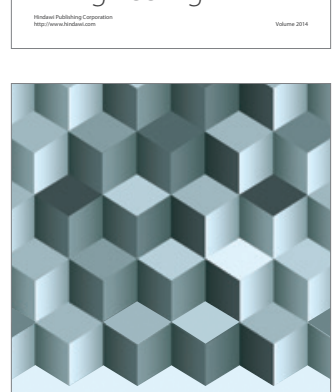

Journal of

Function Spaces
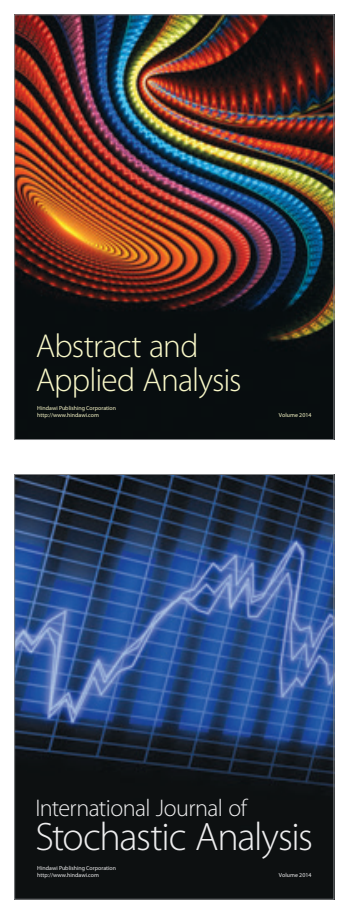

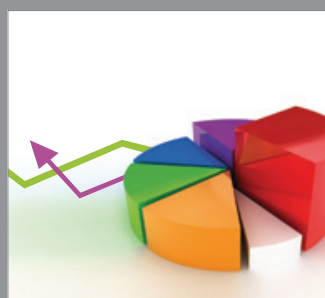

ournal of

Probability and Statistics

Promensencen
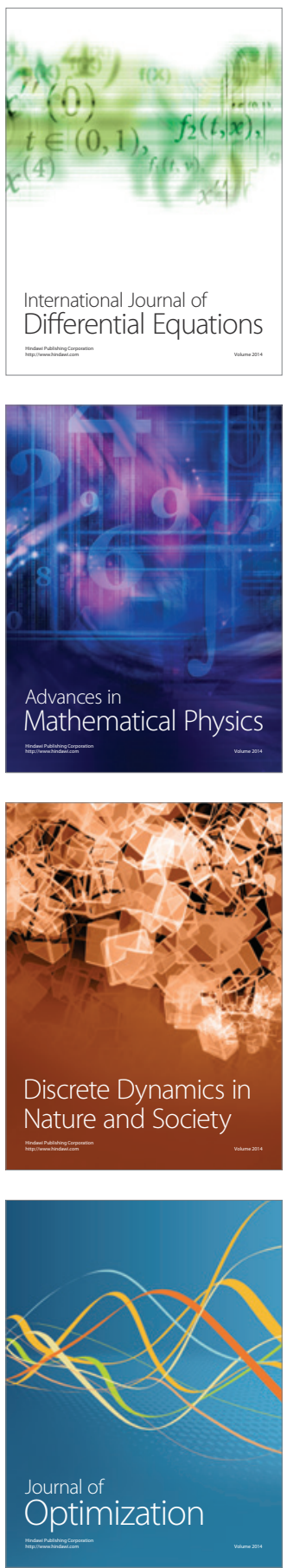